\title{
Spontaneous clearance of Helicobacter pylori after pylorus-preserving gastrectomy for gastric cancer
}

\author{
TOMOHARU MIYASHITA ${ }^{1}$, KOICHI MIWA ${ }^{2}$, MASAFUMI INOKUCHI ${ }^{1}$, HISATOSHI NAKAGAWARA ${ }^{1}$, \\ HIDEHIRO TAJIMA $^{1}$, HIROYUKI TAKAMURA ${ }^{1}$, ITASU NINOMIYA ${ }^{1}$, HIROHISA KITAGAWA ${ }^{1}$, \\ SACHIO FUSHIDA $^{1}$, TAKASHI FUJIMURA ${ }^{1}$, TAKANORI HATTORI ${ }^{3}$ and TETSUO OHTA ${ }^{1}$ \\ ${ }^{1}$ Department of Gastroenterologic Surgery, Kanazawa University Hospital, Kanazawa, Ishikawa 920-8641; \\ ${ }^{2}$ Houju Memorial Hospital, Nomi, Ishikawa 923-1226; ${ }^{3}$ Department of Pathology, \\ Shiga University of Medical Science, Otsu, Shiga 520-2192, Japan
}

Received February 7, 2013; Accepted April 8, 2013

DOI: $10.3892 /$ or.2013.2472

\begin{abstract}
Residual mucosa in the gastric stump after pyloruspreserving gastrectomy (PPG) is considered a risk factor for the development of gastric stump carcinoma(GSC). Duodenogastric reflux (DGR) and Helicobacter pylori (H.pylori) infection are suspected to contribute to the development of GSC. The aim of this study was to investigate the prevalence of $H$. pylori in the residual stomach after PPG for gastric cancer and to assess factors associated with the presence of $H$. pylori. We investigated 72 patients who had undergone PPG at least 1 year prior to the study and were confirmed to be positive for $H$. pylori infection on presurgical endoscopic biopsy. The extent of DGR, the prevalence of $H$. pylori infection based on $H$. pylori stool antigen (HpSA) tests and the severity of gastritis were analyzed in these post-PPG patients. None of the patients had DGR, as shown by ${ }^{99 m}$ Tc-PMT. Of the 72 post-PPG patients, $33(46 \%)$ were positive for HpSA. The prevalence of $H$. pylori infection was significantly lower after surgery than before surgery. The endoscopic severity of remnant gastritis, as well as histological inflammation and activity, were higher in $H$. pylori-positive patients than in $H$. pylori-negative patients. In conclusion, some patients who undergo PPG and are negative for DGR experience spontaneous clearance of $H$. pylori infection.
\end{abstract}

\section{Introduction}

Gastrectomy is a risk factor for the long-term development of gastric stump carcinoma (GSC) (1). The formation of a gastric

Correspondence to: Dr Tomoharu Miyashita, Department of Gastroenterologic Surgery, Kanazawa University Hospital, 13-1 Takaramachi, Kanazawa, Ishikawa 920-8641, Japan

E-mail: tomoharumiya@gmail.com

Abbreviations: H. pylori, Helicobacter pylori; GSC, gastric stump carcinoma; PPG, pylorus-preserving gastrectomy; PPI, Proton pump inhibitor; DGR, duodenogastric reflux

Key words: Helicobacter pylori eradication, pylorus-preserving gastrectomy, gastric stump carcinoma, duodenogastric reflux stump after surgery is considered a precancerous condition (2). Many factors appear to be involved in the etiopathogenesis of GSC, including achlorhydria, hypergastrinemia and biliary reflux, Epstein-Barr virus and Helicobacter pylori (H. pylori) infection, atrophic gastritis, and polymorphisms in the genes encoding interleukin-1 $\beta$ and cyclooxygenase-2 (3-5).

Patients may develop GSC following distal gastrectomy for benign disease. For example, a large population-based study showed that patients who underwent gastric resection for benign disease had an increased risk of cancer in the gastric remnant $\geq 30$ years later (6). In contrast, a small case series showed that patients may develop GCS following distal gastrectomy for cancer $(7,8)$. Early detection of GSC is important, and strict surveillance for a minimum of 10 years has been recommended after initial gastrectomy for gastric cancer $(9,10)$. The incidence of GSC may be higher after the Billroth II procedure than after the Billroth I procedure $(7,11)$, because higher amounts of duodenal contents containing bile persist in the gastric stump after undergoing Billroth II gastrectomy than after Billroth I gastrectomy (12). These findings therefore suggest that duodenogastric reflux (DGR) may be associated with the development of GSC.

$H$. pylori infection is thought to be a significant risk factor for gastric cancer $(13,14)$, with recent epidemiologic evidence suggesting the involvement of $H$. pylori in the carcinogenic process (15). In addition, $H$. pylori eradication has been associated with a reduced likelihood of metachronous cancer development and with tumor growth inhibition, since eradication is associated with the healing of background gastric mucosa (16).

GSC may also be related to $H$. pylori infection. Patients who undergo distal gastric resection have an increased risk of developing GSC, primarily because of DGR. DGR correlates with spontaneous eradication of $H$.pylori infection $(17,18)$, and facilitates the survival of $H$. pylori in the gastric stump, after distal gastrectomy (19).

Proton pump inhibitor (PPI)-based standard therapy is just as effective for eradicating $H$. pylori from the remnant stomach as from the non-surgically treated stomach (20). Eradication therapy results in significant improvements in inflammation and atrophy of the mucosal layer in the remnant stomach after 
early gastric cancer surgery (21). These findings support the role of $H$. pylori in gastric carcinogenesis and suggest that $H$. pylori eradication therapy may prevent the development of metachronous gastric cancer after gastric resection (22).

Pylorus-preserving gastrectomy (PPG), including transectional resection (TR) and local resection (LR), in patients with early gastric cancer along with lymphatic basin dissection, has been found to modulate gastric emptying and prevent DGR $(23,24)$. Moreover, sleeve gastrectomy has been reported to lead to $H$. pylori eradication (25). We investigated the prevalence of $H$. pylori in the residual stomach after PPG for gastric cancer, as well as the correlations between $H$. pylori positivity and the clinical characteristics and severity of gastritis in the residual stomach.

\section{Materials and methods}

Patients. Patients who underwent PPG, including transectional resection (TR) and local resection (LR), at least 1 year prior to this study and were followed up as outpatients in our department were selected for this study. All patients were positive for $H$. pylori infection on endoscopic biopsy before surgery. Subjects taking $\mathrm{H} 2$ receptor antagonists, proton pump inhibitors (PPIs), non-steroidal anti-inflammatory drugs, antibiotics, or bismuth salts, and those who had undergone H. pylori-eradication therapy were excluded. Seventy-two patients agreed to participate in the trial, with all providing prior, written, informed consent. The median patient age was 62 years (range 31-85 years). Forty-eight subjects were male and 24 were female. All patients had undergone PPG for early gastric cancer. The median time from surgery was 5 years (range 1-10 years). None of these patients had experienced local recurrence or metastasis of their original tumor. Two patients developed GSC 9 years after PPG. Of the 72 patients, 46 had undergone TR (23) and 26 had undergone LR; these 2 groups did not differ in regards to patient characteristics, including mean age, gender, distribution, or mean time following surgery.

$D G R$. The degree of DGR was assessed by using hepatobiliary scintigraphy, by monitoring $\mathrm{pH}$, and by endoscopic examination.

Hepatobiliary scintigraphy. Eighteen patients were selected. After an overnight fast, each patient received an intravenous injection of $37 \mathrm{MBq}$ of ${ }^{99} \mathrm{~m} \mathrm{Tc} \mathrm{N}$-pyridoxyl-5methyltryptophan ( ${ }^{99 \mathrm{~m}} \mathrm{Tc}-\mathrm{PMT}$; Japan Medi-Physics, Japan). Patients then underwent serial hepatobiliary scanning in the sitting position using a gamma camera, with images taken at $0,10,20,30,45,60,90$ and $120 \mathrm{~min}$. A region of interest (ROI) corresponding to the remnant stomach was outlined in anterior views, and the radioactivity in each ROI was measured and expressed as a percentage of the radioactivity at time 0 (i.e., reflux amount).

Measurement of $\mathrm{pH}$ in the remnant stomach. Before assessment, the $\mathrm{pH}$ monitor was calibrated, i.e., the tip of the probe was introduced into a liquid and stabilized. The calibrated probe was placed transnasally in the remnant stomach and taped in place, with measurements started after assuring that the equipment was correctly placed. The $\mathrm{pH}$ was measured for $24 \mathrm{~h}$, following which, all data from the device were transferred to a personal computer and analyzed.

Endoscopic examination. All patients underwent endoscopic examination every 6 months following surgery. During upper gastrointestinal endoscopy, performed after an overnight fast, tissue specimens were sampled from the greater curvature of the antrum and the fornix.

Detection of $H$. pylori. Stool samples of all patients were collected and analyzed for $H$. pylori antigen using enzyme immunoassays (HpSA, Premier Platinum HpSA; Meridian Diagnostics Inc., Cincinnati, OH, USA) in accordance with the manufacturer's instructions. Briefly, diluted fecal samples and a peroxidase-conjugated polyclonal antibody were added to the microwells containing polyclonal antibodies to $H$.pylori and incubated for $1 \mathrm{~h}$ at room temperature. The wells were washed to remove unbound materials. Substrate was added and the wells were incubated for $10 \mathrm{~min}$ at room temperature. The presence of bound $H$. pylori antigens was demonstrated by a change in color from blue to yellow. A stop solution was added, and spectrophotometric analysis was performed at $450 \mathrm{~nm}$. Absorbances $<0.140$ were considered negative; those from $0.140-0.159$ were considered equivocal; and those $>0.160$ were considered positive. In these assays, buffer mixed with inactivated $H$. pylori antigen was used as a positive control, and buffer mixed with preservative was used as a negative control.

Histological assessment of biopsy specimens. Biopsy specimens from each site of the stomach were oriented on filter paper and immediately fixed in $10 \%$ buffered formalin. Paraffin-processed sections were cut at 3 levels and stained with hematoxylin and eosin (H\&E). The sections were examined in a blinded manner by a single pathologist and specifically assessed for severity of gastritis using the updated Sydney system. The degree of inflammation, activity, atrophy and intestinal metaplasia at each site was graded as normal, mild, moderate or severe.

Statistical analysis. Differences between groups were analyzed using the Student's t-test, the Mann-Whitney rank sum test, Fisher's exact test, or the log-rank test, as appropriate. All statistical analyses were performed using StatView software (SAS Co., Berkeley, CA, USA), with p-value $<0.05$ considered to indicate a statistically significant result.

Ethics. The study was performed according to the Declaration of Helsinki and approved by the Regional Ethics Committee of Kanazawa University.

\section{Results}

Environment of the remnant stomach after $P P G$. Of the 18 patients tested, none showed detectable ${ }^{99 \mathrm{~m}} \mathrm{Tc}-\mathrm{PMT}$ in the remnant stomach, indicating an absence of bile reflux in patients who had undergone PPG at least 1 year earlier. Representative findings of ${ }^{99 \mathrm{~m}} \mathrm{Tc}-\mathrm{PMT}$ and $\mathrm{pH}$ monitoring in a post-LR patient showed no DGR and a mean intragastric $\mathrm{pH}$ of 5.2 over $24 \mathrm{~h}$ (Fig. 1). 

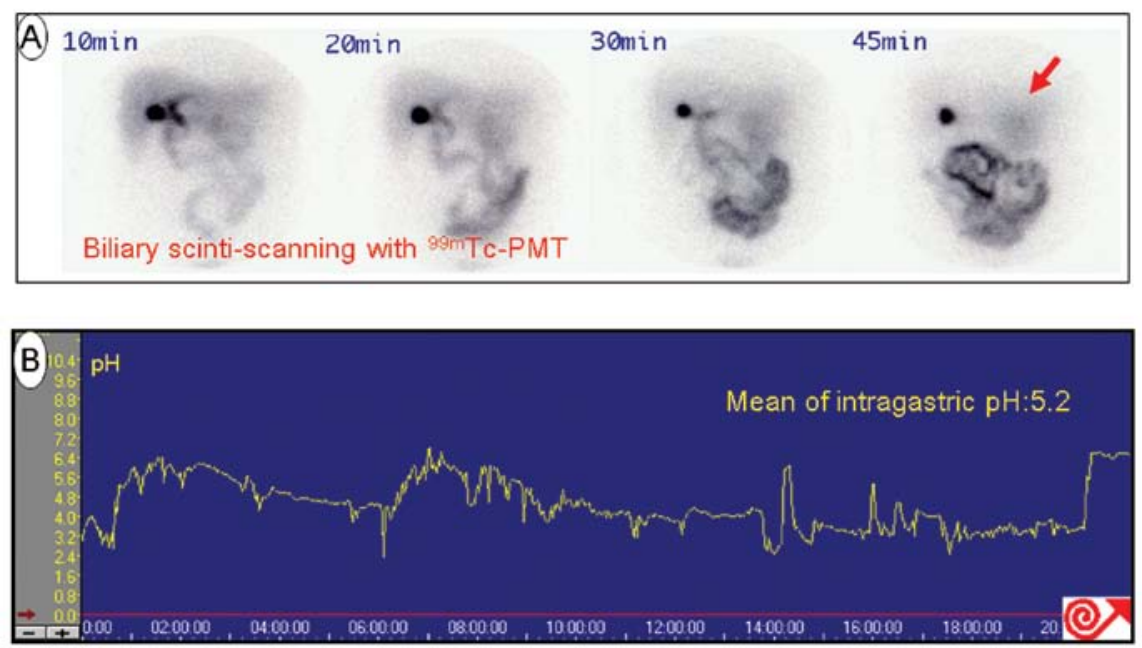

Figure 1. Scintigraphy and $\mathrm{pH}$ monitoring in a representative patient. (A) ${ }^{99 \mathrm{~m}} \mathrm{Tc}-\mathrm{PMT}$ showing a complete absence of duodenogastric reflux (DGR). Red arrow indicates remnant stomach. (B) Measurement of intragastric $\mathrm{pH}$ over $24 \mathrm{~h}$, with a mean intragastric $\mathrm{pH}$ of 5.2.

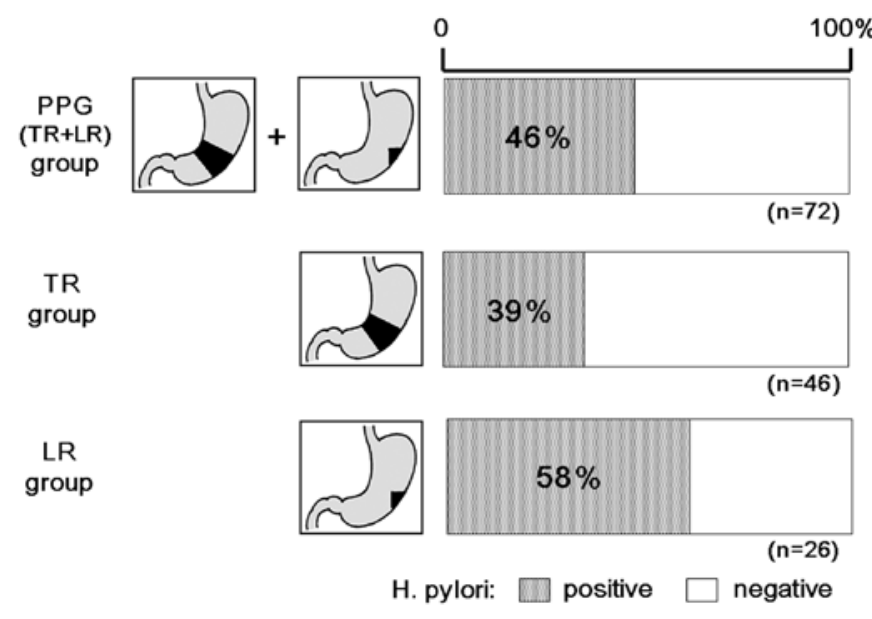

Figure 2. Incidence of $H$. pylori infection before and after pylorus-preserving gastrectomy (PPG). All patients were confirmed as being H. pylori infectionpositive on endoscopic biopsy before surgery. The prevalence of $H$. pylori infection was significantly reduced after PPG, whether transectional resection (TR) or local resection (LR).

Upper gastrointestinal endoscopy showed that 2 of the $72(3 \%)$ patients had mild DGR.

Presence of $H$. pylori. Of the 72 patients, 33 (46\%) were positive for HpSA, including 18 of the 46 (39\%) patients who had undergone TR and 16 of the $26(58 \%)$ patients who had undergone LR. The overall prevalence of $H$. pylori infection was significantly lower after PPG than before PPG (Fig. 2).

Degree of gastritis. We assessed the relationship between $H$. pylori infection and the endoscopic severity of remnant gastritis. Endoscopically, we observed redness and edema throughout the remnant gastric mucosa, with the incidence of both being higher in $H$. pylori-positive than in $H$. pylori-negative patients. Moreover, histological evaluation of the biopsy specimens taken from the greater curvature of the antrum and the fornix showed that both inflammation and activity were

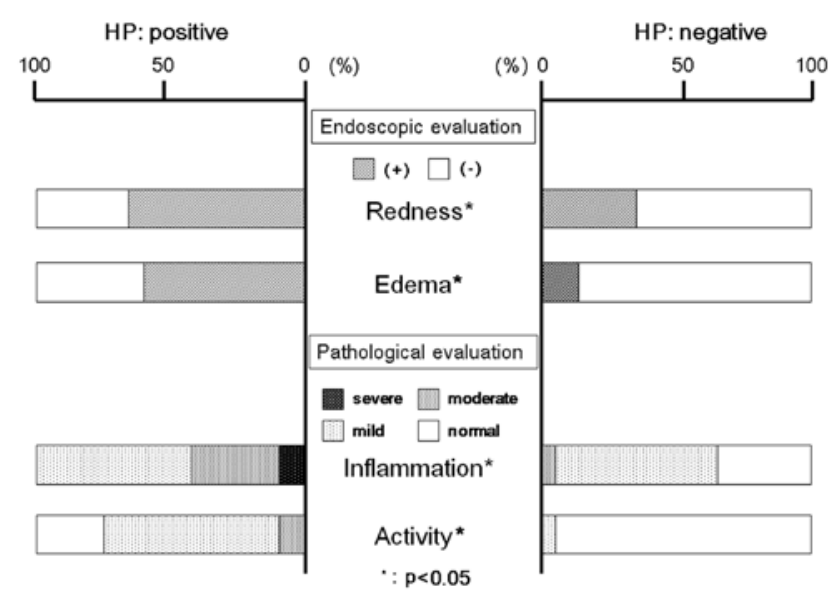

Figure 3. Associations between endoscopic and histological findings with $H$. pylori infection in the remnant stomach. The rates of redness, edema, inflammation and activity were significantly higher in the post-pyloruspreserving gastrectomy (PPG) mucosae of $H$. pylori-positive patients than in the post-PPG mucosae of $H$. pylori-negative patients.

higher in $H$. pylori-positive than in the $H$. pylori-negative patients (Fig. 3).

Outcomes in post-PPG patients with and without H. pylori infection. We found that 2 patients had GSC with persistent H. pylori infection 9 years after PPG (Fig. 4A and B), but both were negative for bile reflux using ${ }^{99 \mathrm{~m}} \mathrm{Tc}-\mathrm{PMT}$.

Of the 33 patients positive for $H$. pylori after PPG, 6 underwent $H$. pylori eradication using standardized methods, with eradication in all 6 being successful. One of these 6 patients had an ulcer, and another had reflux esophagitis after eradication (Fig. 4C and D), but both were successfully treated with PPIs.

\section{Discussion}

To the best of our knowledge, this is the first study to compare the spontaneous reduction in $H$. pylori infection and the 


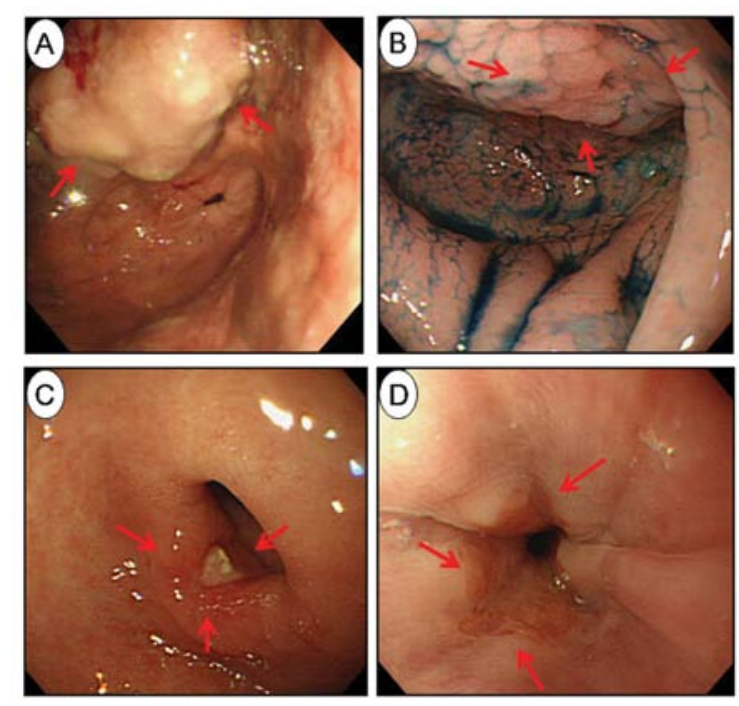

Figure 4. Complications in patients after pylorus-preserving gastrectomy (PPG). (A and B) Two patients with residual gastric cancer and persistent H. pylori infection 9 years after PPG. (C) One patient had an ulcer after eradication. (D) One patient had GERD after eradication.

presence of mucosal lesions in the remnant stomach after PPG for gastric cancer.

The presence of residual mucosa in the gastric stump is considered a risk factor for GSC, as well as a precancerous condition, independent of indications for surgery (22). Although there are more sensitive methods for detecting $H$. pylori, we assessed $H$. pylori infection status using stool HpSA tests. The use of stool tests may explain the lower prevalence of $H$. pylori infection among our patients than in a previous study (26). However, bacterial culture and histopathological examinations are direct but more invasive methods, whereas the urea breath and stool HpSA tests are both indirect and non-invasive methods of detecting $H$. pylori (27). Comparisons of urease and stool HpSA tests with histopathology showed that the sensitivity and specificity of the urease tests were 62.2 and $100 \%$ respectively, whereas the sensitivity and specificity of the stool tests were 68.9 and $100 \%$ respectively, indicating that the results obtained with biopsy urease and HpSA tests were generally similar (28), and that stool HpSA tests are generally useful for the diagnosis of $H$. pylori infection.

Because it constitutes a precancerous condition, the resected stomach offers an opportunity to assess the factors involved in gastric carcinogenesis. Bile reflux interferes with the prevalence of $H$. pylori infection $(17,18)$. Roux-en-Y reconstruction following distal gastrectomy has been shown to be superior to Billroth I and II reconstruction in preventing remnant gastritis, as the former reduces DGR (29). Therefore, Roux-en-Y reconstruction may reduce $H$. pylori infection by preventing bile reflux and gastritis (30). The prevalence of $H$. pylori infection was found to be low following jejunal pouch interposition and Roux-en-Y reconstruction, which prevent bile reflux, but high in patients who underwent Billroth I and II reconstruction. DGR was rare in patients who underwent jejunal pouch interposition and Roux-en-Y reconstruction, but was often observed in patients who underwent Billroth I and II reconstruction, suggesting a positive relationship between DGR and H.pylori infection (19). We found that the PPG procedure prevented bile reflux, as shown by ${ }^{99 \mathrm{~m}} \mathrm{Tc}-\mathrm{PMT}$, and reduced the prevalence of $H$. pylori significantly when compared with its prevalence prior to PPG, providing further evidence of the positive relationship between DGR and $H$.pylori infection.

Routine post-surgical treatment with the antibiotic cephalosporin may affect $H$. pylori infection. However, an evaluation of $8 \mathrm{H}$. pylori-positive colorectal cancer patients showed that none became $H$. pylori-negative after surgery and antibiotic treatment. These findings suggest that post-operative antibiotic treatment is not associated with eradication of $H$. pylori infection after surgery.

We found that 2 patients had residual gastric cancer with persistent $H$. pylori infection 9 years after PPG. Of the 33 H.pylori-positive patients after PPG, 6 underwent $H$.pylori eradication using standardized methods, with eradication in all 6 being successful. One of these patients had GERD, and another had an ulcer after eradication, but both were successfully treated with PPIs, suggesting that eradication therapy may prevent GCS. PPI-based therapy was as effective in eradication $H$. pylori in remnant stomachs as in unoperated stomachs, with eradication therapy significantly decreasing inflammatory cell infiltration of the mucosal layer $(20,31)$. In addition, eradication therapy decreased the Ki-67 labeling index and almost normalized tissue IL-8 levels, suggesting that $H$. pylori eradication may reduce the risk of $H$. pyloriassociated carcinogenesis in patients who have undergone gastrectomy for early gastric cancer (32).

Our findings suggest that PPG itself may lead to H.pylori eradication. Further clinical studies on larger populations are needed to address this issue and to formulate appropriate guidelines for this relatively new procedure.

\section{References}

1. Sinning C, Schaefer N, Standop J, Hirner A and Wolff M: Gastric stump carcinoma - epidemiology and current concepts in pathogenesis and treatment. Eur J Surg Oncol 33: 133-139, 2007.

2. van Rees BP, Saukkonen K, Ristimäki A, et al: Cyclooxygenase-2 expression during carcinogenesis in the human stomach.J Pathol 196: 171-179, 2002.

3. Sitarz R, Maciejewski R, Polkowski WP and Offerhaus GJ: Gastroenterostoma after Billroth antrectomy as a premalignant condition. World J Gastroenterol 18: 3201-3206, 2012.

4. Zur Hausen A, van Rees BP, van Beek J, et al: Epstein-Barr virus in gastric carcinomas and gastric stump carcinomas: a late event in gastric carcinogenesis. J Clin Pathol 57: 487-491, 2004.

5. Johannesson KA, Hammar E and Staël von Holstein C: Mucosal changes in the gastric remnant: long-term effects of bile reflux diversion and Helicobacter pylori infection. Eur J Gastroenterol Hepatol 15: 35-40, 2003.

6. Lagergren J, Lindam A and Mason RM: Gastric stump cancer after distal gastrectomy for benign gastric ulcer in a population-based study. Int J Cancer 131: E1048-E1052, 2012.

7. Sowa M, Onoda N, Nakanishi I, et al: Early stage carcinoma of the gastric remnant in Japan. Anticancer Res 13: 1835-1838, 1993.

8. Kaminishi M, Shimizu N, Yamaguchi H, Hashimoto M, Sakai S and Oohara T: Different carcinogenesis in the gastric remnant after gastrectomy for gastric cancer. Cancer 77: 1646-1653, 1996.

9. Ohashi M, Katai H, Fukagawa T, Gotoda T, Sano T and Sasako M: Cancer of the gastric stump following distal gastrectomy for cancer. Br J Surg 94: 92-95, 2007.

10. Kaneko K, Kondo H, Saito D, et al: Early gastric stump cancer following distal gastrectomy. Gut 43: 342-344, 1998.

11. Thorban S, Böttcher K, Etter M, Roder JD, Busch R and Siewert JR: Prognostic factors in gastric stump carcinoma. Ann Surg 231: 188-194, 2000. 
12. Lorusso D, Linsalata M, Pezzolla F, et al: Duodenogastric reflux and gastric mucosal polyamines in the non-operated stomach and in the gastric remnant after Billroth II gastric resection. A role in gastric carcinogenesis? Anticancer Res 20: 2197-2201, 2000.

13. Goodwin CS, Armstrong JA and Marshall BJ: Campylobacter pyloridis, gastritis, and peptic ulceration. J Clin Pathol 39: 353-365, 1986.

14. Matsumoto Y, Marusawa H, Kinoshita K, et al: Helicobacter pylori infection triggers aberrant expression of activationinduced cytidine deaminase in gastric epithelium. Nat Med 13: 470-476, 2007.

15. Takahashi S: Long-term Helicobacter pylori infection and the development of atrophic gastritis and gastric cancer in Japan. J Gastroenterol 37 (Suppl 13): 24-27, 2002.

16. Uemura $\mathrm{N}$ and Okamoto S: Effect of Helicobacter pylori eradication on subsequent development of cancer after endoscopic resection of early gastric cancer in Japan. Gastroenterol Clin North Am 29: 819-827, 2000

17. Fukuhara K, Osugi H, Takada N, et al: Duodenogastric reflux eradicates Helicobacter pylori after distal gastrectomy. Hepatogastroenterology 51: 1548-1550, 2004.

18. Abe H, Murakami K, Satoh S, et al: Influence of bile reflux and Helicobacter pylori infection on gastritis in the remnant gastric mucosa after distal gastrectomy. J Gastroenterol 40: 563-569, 2005.

19. Nakagawara H, Miwa K, Nakamura S and Hattori T: Duodenogastric reflux sustains Helicobacter pylori infection in the gastric stump. Scand J Gastroenterol 38: 931-937, 2003.

20. Matsukura N, Tajiri T, Kato S, et al: Helicobacter pylori eradication therapy for the remnant stomach after gastrectomy. Gastric Cancer 6: 100-107, 2003.

21. Kato S, Matsukura N, Matsuda N, Tsuchiya S, Naito Z and Tajiri T: Normalization of $\mathrm{pH}$ level and gastric mucosa after eradication of $H$. pylori in the remnant stomach. J Gastroenterol Hepatol 23 (Suppl 2): S258-S261, 2008

22. Giuliani A, Galati G, Demoro M, Scimò M, Pecorella I and Basso L: Screening of Helicobacter pylori infection after gastrectomy for cancer or peptic ulcer: results of a cohort study. Arch Surg 145: 962-967, 2010.
23. Fujimura T, Fushida S, Kayahara M, Ohta T, Kinami S and Miwa K: Transectional gastrectomy: an old but renewed concept for early gastric cancer. Surg Today 40: 398-403, 2010.

24. Kitagawa Y and Kitajima M: Diagnostic validity of radio-guided sentinel node mapping for gastric cancer: a review of current status and future direction. Surg Technol Int 15: 32-36, 2006.

25. Keren D, Matter I, Rainis T, Goldstein O, Stermer E and Lavy A: Sleeve gastrectomy leads to Helicobacter pylori eradication. Obes Surg 19: 751-756, 2009.

26. Deguchi R, Matsushima M, Suzuki T, et al: Comparison of a monoclonal with a polyclonal antibody-based enzyme immunoassay stool test in diagnosing Helicobacter pylori infection after eradication therapy. J Gastroenterol 44: 713-716, 2009.

27. Hooton C, Keohane J, Clair J, et al: Comparison of three stool antigen assays with the 13C-urea breath test for the primary diagnosis of Helicobacter pylori infection and monitoring treatment outcome. Eur J Gastroenterol Hepatol 18: 595-599, 2006.

28. Ceken N, Yurtsever SG, Baran N, Alper E, Buyrac Z and Unsal B: Comparison of Helicobacter pylori antibody detection in stool with other diagnostic tests for infection. Asian Pac J Cancer Prev 12: 1077-1081, 2011.

29. Osugi H, Fukuhara K, Takada N, Takemura M and Kinoshita H: Reconstructive procedure after distal gastrectomy to prevent remnant gastritis. Hepatogastroenterology 51: 1215-1218, 2004.

30. Chan DC, Fan YM, Lin CK, Chen CJ, Chen CY and Chao YC: Roux-en-Y reconstruction after distal gastrectomy to reduce enterogastric reflux and Helicobacter pylori infection. J Gastrointest Surg 11: 1732-1740, 2007.

31. Onoda N, Katsuragi K, Sawada T, et al: Efficacy of Helicobacter pylori eradication on the chronic mucosal inflammation of the remnant stomach after distal gastrectomy for early gastric cancer. J Exp Clin Cancer Res 24: 515-521, 2005.

32. Hamaguchi K, Ogawa K, Katsube T, Konno S and Aiba M: Does eradication of Helicobacter pylori reduce the risk of carcinogenesis in the residual stomach after gastrectomy for early gastric cancer? Comparison of mucosal lesions in the residual stomach before and after Helicobacter pylori eradication. Langenbecks Arch Surg 389: 83-91, 2004. 